\title{
Paisajes construidos con ayuda de Geometría analítica y con GeoGebra
}

\author{
Alberto Guadarrama Herrera ${ }^{1}$, Fernando Becerril Morales ${ }^{2}$, Joel Díaz Silva ${ }^{3}$ \\ 1,2,3 Plantel Dr. "Pablo González Casanova" de la Universidad Autónoma del Estado de México, UAEMéx. - C.P. \\ 52400-Tenancingo, Estado de México, México. \\ aguadarramah@uaemex.mx, fbecerrilm@uaemex.mx, jdiazs@uaemex.mx
}

\begin{abstract}
The strategy is aimed at third-year high school students. The activity to be carried out consists of an unpublished landscape that contains lines, circumferences, parabolas, ellipses and / or hyperbolas, to be constructed with the help of GeoGebra.

The student from his imagination pours the idea into a sketch and during the whole activity develops the following aspects: the creative part, the analytical part when migrating the idea into equations of the different conics, thereby strengthening the mathematical ability and skill in the use of ICT with the use of GeoGebra. By carrying out projects of this nature, it allows the teacher a renewal in the activities of the subject and the students generate greater interest in mathematics.
\end{abstract}

Resumen. La estrategia está dirigida a estudiantes de tercer semestre de bachillerato. La actividad a desarrollar consiste en construir, con la ayuda de GeoGebra, un paisaje inédito que contenga rectas, circunferencias, parábolas, elipses y/o hipérbolas.

El estudiante desde su imaginación vierte la idea en un boceto y durante toda la actividad desarrolla los siguientes aspectos: la parte creativa, la parte analítica al migrar la idea en ecuaciones de las diferentes cónicas, con ello fortalece la habilidad matemática y la destreza en el uso de las TIC con el uso de GeoGebra. Al realizar proyectos de esta indole permite al docente una renovación en las actividades propias de la asignatura y a los alumnos les genera mayor interés hacia las matemáticas.

\section{Introducción}

Los procesos de aprendizaje basados en competencias responden a diversas necesidades, por tanto es necesario incluir en la educación en México una formación holística que permita integrar tanto la teoría como la práctica en un contexto real y aplicable en su vida cotidiana. Todo ello con el propósito de fortalecer los niveles de aprendizaje y mejorar la calidad educativa, que tiene como objetivo primordial la disminución de la deserción escolar y la mejora en las estadísticas a nivel nacional e internacional como son las pruebas Enlace, Exani, Excale o PISA 
VIII Congresso Brasileiro de Informática na Educação (CBIE 2019)

Anais dos Workshops do VIII Congresso Brasileiro de Informática na Educação (WCBIE 2019)

instrumentados por la OCDE (Organización para la Cooperación y Desarrollo Económico) en nuestro país.

Es por ello que la Universidad Autónoma del Estado de México (UAEMéx), ha venido implementando diversos cambios en sus programas de estudio para afrontar la mejora en educación en el Nivel Medio Superior, con base a sus preceptos básicos que corresponde a el logro de una formación humanista, comunicativa, crítico-intelectual, social y para la vida, además de científica y tecnológica (UAEM, 2009) en sus egresados, que se vea reflejado en la responsabilidad, participación, colaboración, ética, entre otros; que corresponde a las diversas dimensiones de formación que muestra el perfil de egreso. Teniendo presente que el fin primordial de las instituciones que conforman el NMS corresponde a la continuidad de los estudios en el Nivel Superior de sus egresados (UAEM, 2009). Realizando las adecuaciones pertinentes en el currículum universitario tanto en su contenido como en su vinculación con las diversas disciplinas, todo este trabajo desarrollado conforme a la Reforma Integral para la Educación en el nivel Medio Superior (RIEMS)

\section{Fundamentos teóricos}

El proceso de enseñanza debe de estar fundamentado en la construcción de su conocimiento por medio de una estrategia de enseñanza conjunta entre docente, estudiante y contexto que permita la relación de contenidos mediante una secuencia o programa, con el fin de lograr el procesamiento de la información de un nivel básico a un multi-estructural y relacional, como es mencionado en la Taxonomía SOLO (Structure of the Observed Learning Outcome) [Biggs, 2006]. Para ello se pretende la inclusión de una actividad de aprendizaje y recursos didácticos, que permita la evaluación de los conocimientos, habilidades, actitudes y valores, además de información acorde a la actualidad imperante en nuestro país.

La asignatura de geometría analítica que se imparte en el tercer semestre del nivel medio superior (NMS), tiene como objetivo de la asignatura: desarrollar conocimientos, habilidades, destrezas y actitudes en el alumno para que emplee algunos elementos de la Geometría Analítica que le permitan resolver de manera efectiva situaciones reales, hipotéticas o formales cuyo modelo sea una recta y/o una cónica.

El Propósito de la asignatura es: desarrollar las habilidades, destrezas y actitudes para conocer y emplear los diferentes elementos de la Geometría Analítica en la resolución de problemas al utilizar el lenguaje, los conceptos y principios básicos que le permiten construir representaciones, conceptos y objetos de su entorno que se modelan a través de la elipse e hipérbola en diferentes situaciones de contexto.

Con base en los objetivos plasmados se aplica la estrategia de enseñanza denominada codificación (elaborativa) de la información a aprender [Díaz y Hernández, 2002], que permite la unión de aprendizajes previos en conjunto con la codificación, que permite fusionar las fórmulas aprendidas en periodos anteriores para ser incluida en un programa que le permita ver su aplicación de las diversas figuras como son rectas, parábolas, circunferencia y elipse que permiten al estudiante la aplicación de la geometría analítica en situaciones contextuales logrando enriquecer su asimilación de información nueva mediante gráficos.

La codificación (elaborativa) permite atraer la atención de los estudiantes mediante el uso de un software dinámico llamado GeoGebra, el uso de las tecnologías de la información y comunicación (TIC) permiten una mejor interacción y actuación del alumno en su conocimiento para el logro de un conocimiento básico a un extendido al ubicar en un proceso de elaboración y visualización de la información que se ubican en el proceso al seleccionar, detectar, seguir un 
VIII Congresso Brasileiro de Informática na Educação (CBIE 2019)

Anais dos Workshops do VIII Congresso Brasileiro de Informática na Educação (WCBIE 2019)

proceso y resolver un problema logrando un proceso multiestructural hasta relacional al concretar una actividad con diversas fórmulas relacionando temas anteriores y actuales mediante la aplicación de un programa.

Con ayuda de la algorítmica [Díaz y Hernández, 2002], que apoya a la codificación mediante la descripción de procedimientos para su aplicación y solución de problemas, al obtener su producto del tema de manera visual al favorecer el recordar las fórmulas, su presentación gráfica y no mostrar de manera fragmentada lo teórico con lo práctico, siendo una forma de organizador y soporte de construcción mental en el estudiante.

Al elaborar un paisaje inédito que contenga las diferentes cónicas (rectas, circunferencias, parábolas, elipses y/o hipérbolas) empleando para ello GeoGebra.

\section{Metodología}

El propósito de realizar una investigación-acción [Álvarez y Gayou, 2003] en el ámbito académico, es para resolver problemáticas cotidianas, es por ello que la estrategia de aprendizaje de codificación en el GeoGebra, interviene de manera normal en el proceso de enseñanza-aprendizaje permitiendo involucrar a los estudiantes en una dinámica diferente para su desarrollo académico. Mediante el modelo de enseñanza basado en problemas, como a continuación se explica.

En la asignatura de Geometría Analítica, tradicionalmente la forma de trabajo era resolver los diferentes ejercicios, los cuales tienen ciertas condiciones iniciales, por ejemplo: coordenadas, pendientes, intersección con los ejes, etc. De la información anterior, se desarrolla cierto procedimiento para finalmente representarlo en una gráfica en el plano cartesiano.

Para esta estrategia didáctica, la idea central es invertir el proceso anterior, el cual consiste en partir de una serie de gráficos (paisaje elaborado por el alumno) y obtener las diferentes ecuaciones de cada uno de los elementos que la conforman, para posteriormente construir con GeoGebra.

El uso del software permite la interacción entre la teoría y la práctica a desarrollar en la temática el curso, las características de GeoGebra, que es un software libre y el cual está diseñado de tal manera que es muy amigable para ser manipulado, puede ser descargado de la siguiente página: https://www.geogebra.org/.

Por otro lado, toda la actividad se trabaja a lo largo un semestre completo, una vez por semana se revisan avances del proyecto, con la finalidad de que los alumnos vayan conociendo el ambiente de trabajo con el software, la idea es trabajar de manera individual, pero esto en ocasiones no es posible por el número de integrantes del grupo (ver figura 1). 
VIII Congresso Brasileiro de Informática na Educação (CBIE 2019)

Anais dos Workshops do VIII Congresso Brasileiro de Informática na Educação (WCBIE 2019)

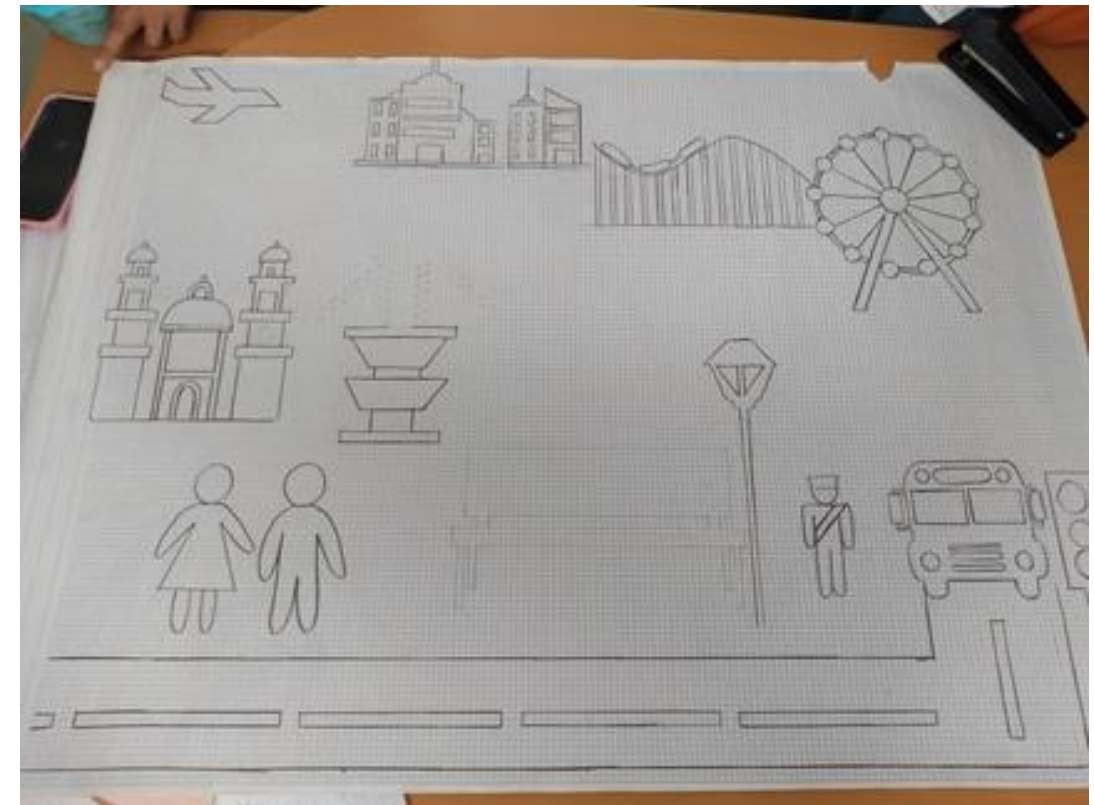

Figura 1. Revisión del primer avance del proyecto

En las primeras sesiones de adiestramiento del paquete computacional mencionado, se les sugiere a los estudiantes que tomen nota de cómo introducir los datos (ecuaciones), así como las restricciones que se pueden colocar para solo trazar una parte de la gráfica, por consiguiente, se explican todas las herramientas posibles que en algún momento utilizarán para elaborar cada paisaje. (Ver Figura 2)

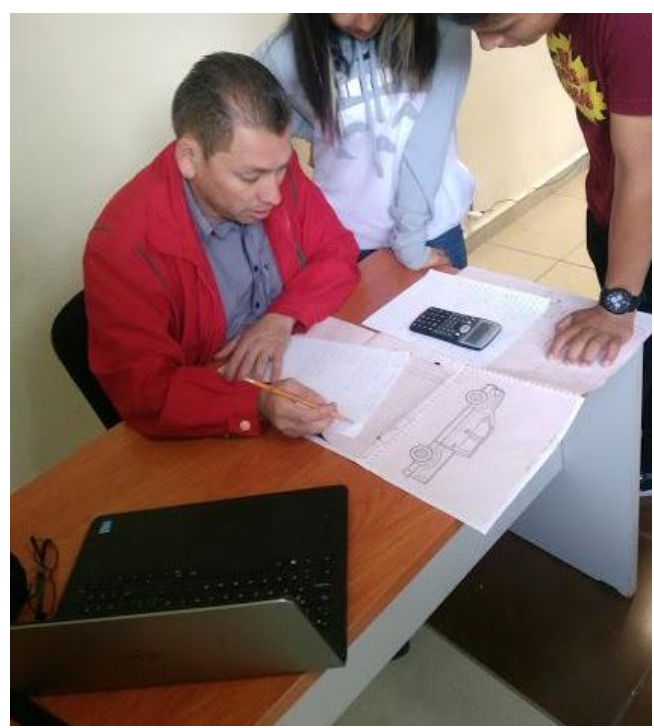

Figura 2. Asesoría de cómo introducir las ecuaciones a GeoGebra.

Es importante mencionar que para el desarrollo de esta estrategia didáctica no es posible llevarla en una sesión de $50 \mathrm{~min}$, por el contrario, se requieren de varias sesiones, teniendo escenarios distintos como: el aula, la sala de computación o el domicilio de cada estudiante. 
VIII Congresso Brasileiro de Informática na Educação (CBIE 2019)

Anais dos Workshops do VIII Congresso Brasileiro de Informática na Educação (WCBIE 2019)

A continuación, se enuncian una serie de pasos que se requieren para desarrollar la estrategia didáctica:

1. Por equipo se solicita un boceto del paisaje a elaborar. Cada integrante realizará una parte en una hoja milimétrica o en su defecto en una hoja de cuadro chico.

2. El profesor revisa cada boceto e indica a los integrantes las modificaciones pertinentes a realizar en caso que no puedan ser graficados en GeoGebra.

3. Una vez corregido y aprobado cada boceto, los integrantes deberán obtener todos los datos necesarios para la obtención de las diferentes ecuaciones, iniciando con las coordenadas de los elementos esenciales de cada cónica.

4. El alumno con dichos elementos y con la ayuda de las expresiones matemáticas correspondientes determina cada una de las ecuaciones de las diferentes canónicas que darán vida al paisaje.

5. En algunos casos, solo se requiere trazar una porción de la gráfica, es aquí donde el alumno tendrá que ingresar una limitante, en matemáticas se le conoce como dominio de la función, la cual son los límites a lo largo del eje "x".

6. Como no se tiene la habilidad suficiente para tener la o las ecuaciones correctas a la primera, se sugiere anotar en su cuaderno aquellas que le sirvan. Ya que se está trabajando bajo un esquema de prueba y error.

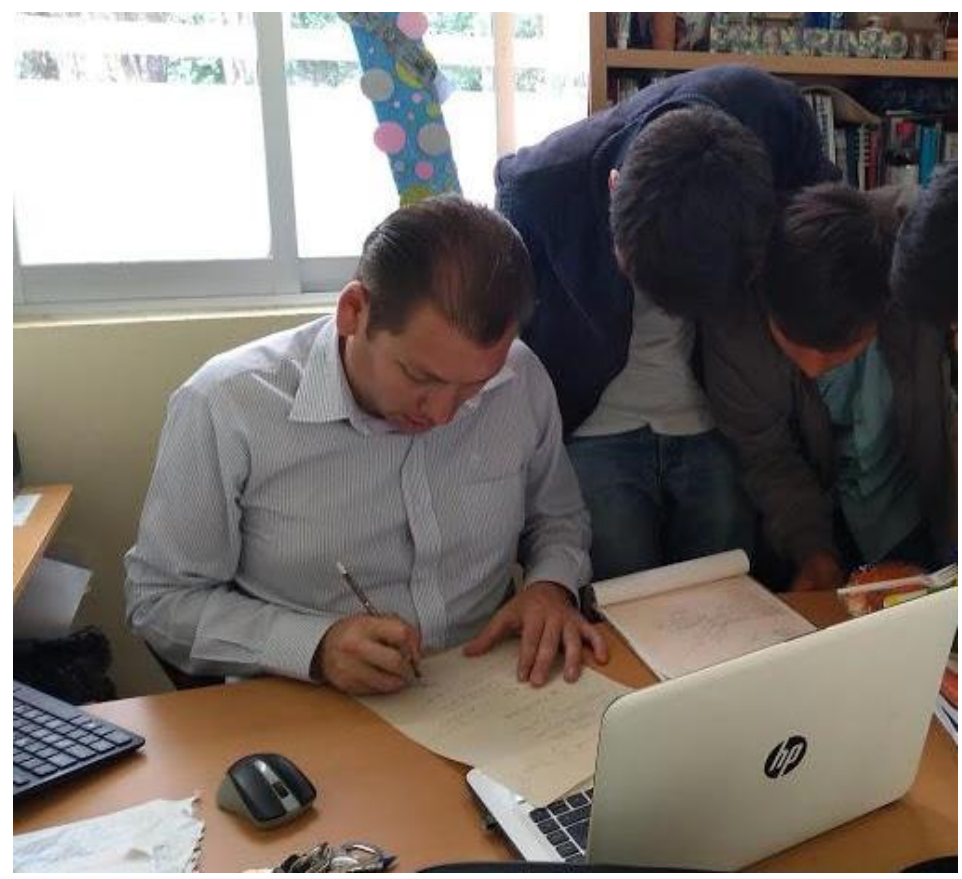

Figura 3. Explicando cómo delimitar ecuaciones en GeoGebra

7. Al tener todas las ecuaciones necesarias para la elaboración de la figura, se sugiere al alumno, abrir una hoja nueva de trabajo, e ingresar cada ecuación hasta la totalidad de las mismas.

8. Una vez terminado de ingresar todas las ecuaciones, se guarda y se manda a imprimir. 
VIII Congresso Brasileiro de Informática na Educação (CBIE 2019)

Anais dos Workshops do VIII Congresso Brasileiro de Informática na Educação (WCBIE 2019)

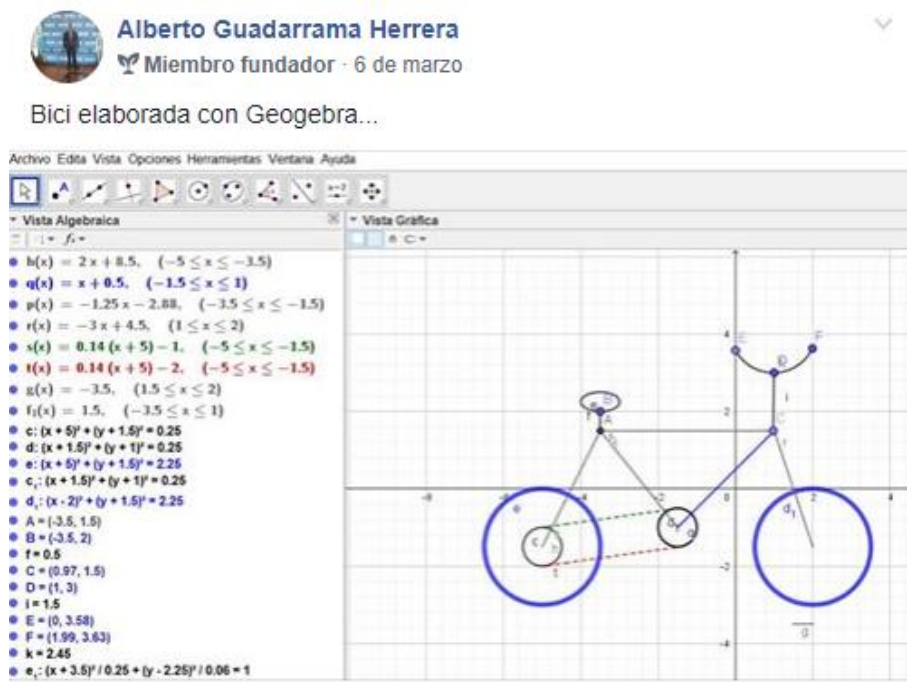

Figura 4. Bicicleta elaborada con GeoGebra

9. Se envía el proyecto final al correo electrónico del profesor de la asignatura.

10. Por último, se expone en plenaria dentro del salón de clase o en un espacio alterno, invitando a la demás comunidad del espacio académico, incluyendo a los docentes que imparten la materia en otros grupos. En esta sesión se les pide a los alumnos manifestar sus experiencias generadas a lo largo del desarrollo de toda esta estrategia didáctica.

\section{Resultados y Conclusiones}

Esta estrategia didáctica se ha trabajado desde el año 2009, iniciando con 4 grupos, creciendo año con año, actualmente (periodo febrero-julio 2019), se tuvo la participación de 16 grupos en total.

En todos los casos se ha tenido diferentes niveles de éxito, que van desde aquellos que no lograron comprender la finalidad de la actividad en sus diferentes etapas; transitando por aquellos casos en los que el nivel va creciendo conforme se desarrolla el proyecto; hasta llegar a los alumnos destacados que le entienden desde inicio, son constantes en la elaboración, así como en acudir a las diferentes asesorías con el docente. Este último grupo de estudiantes nos ayudan a ser monitores en los diferentes grupos. 
VIII Congresso Brasileiro de Informática na Educação (CBIE 2019)

Anais dos Workshops do VIII Congresso Brasileiro de Informática na Educação (WCBIE 2019)

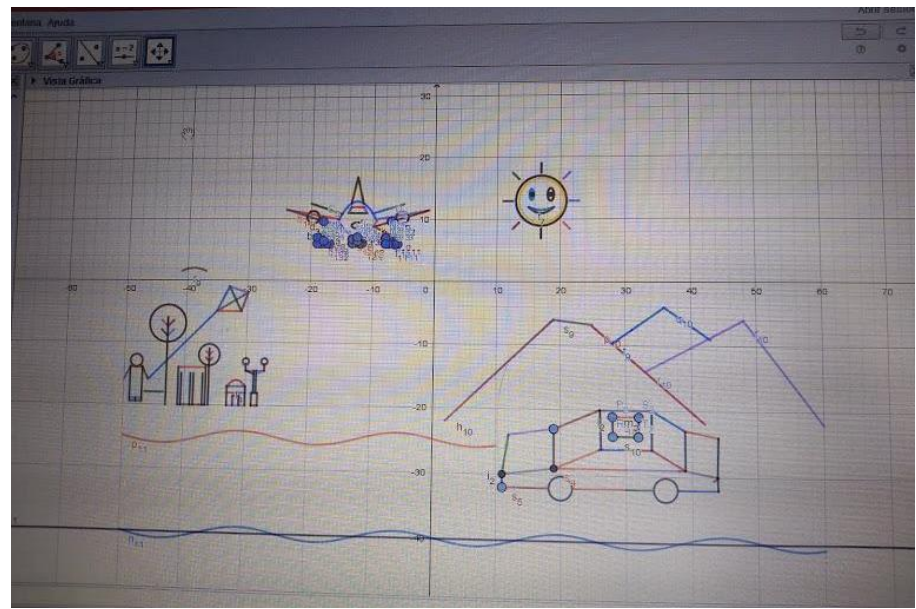

Figura 5. Proyecto Terminado

Es muy importante destacar que gracias a las diferentes asesorías por parte de un servidor o de los alumnos que fungen como mentores académicos, permiten que el $85 \%$ del grupo, tenga éxito.

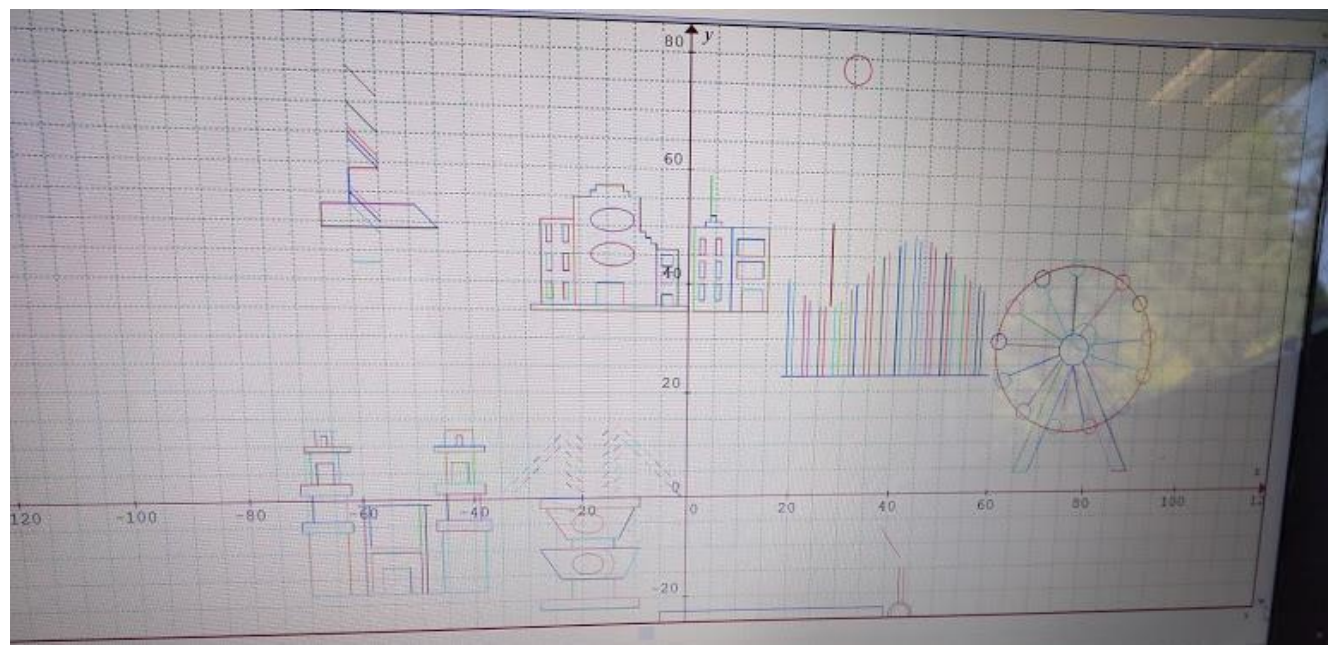

Figura 6. Proyecto Terminado

Toda estrategia varía en cada grupo, el éxito no está siempre asegurado, incluso si no se trabaja correctamente, esta estrategia se puede convertir en un fracaso. Uno como docente debe estar preparado para realizar los ajustes necesarios a la secuencia de la actividad.

En esta actividad, la transversalidad queda plasmada en cada trabajo elaborado por los estudiantes, así mismo, se ha logrado desarrollar su creatividad a través de la aplicación de las matemáticas - lo que es altamente gratificante - en el proceso de enseñanza aprendizaje, lo cual contribuye significativamente a su formación y crecimiento académico. 
VIII Congresso Brasileiro de Informática na Educação (CBIE 2019)

Anais dos Workshops do VIII Congresso Brasileiro de Informática na Educação (WCBIE 2019)

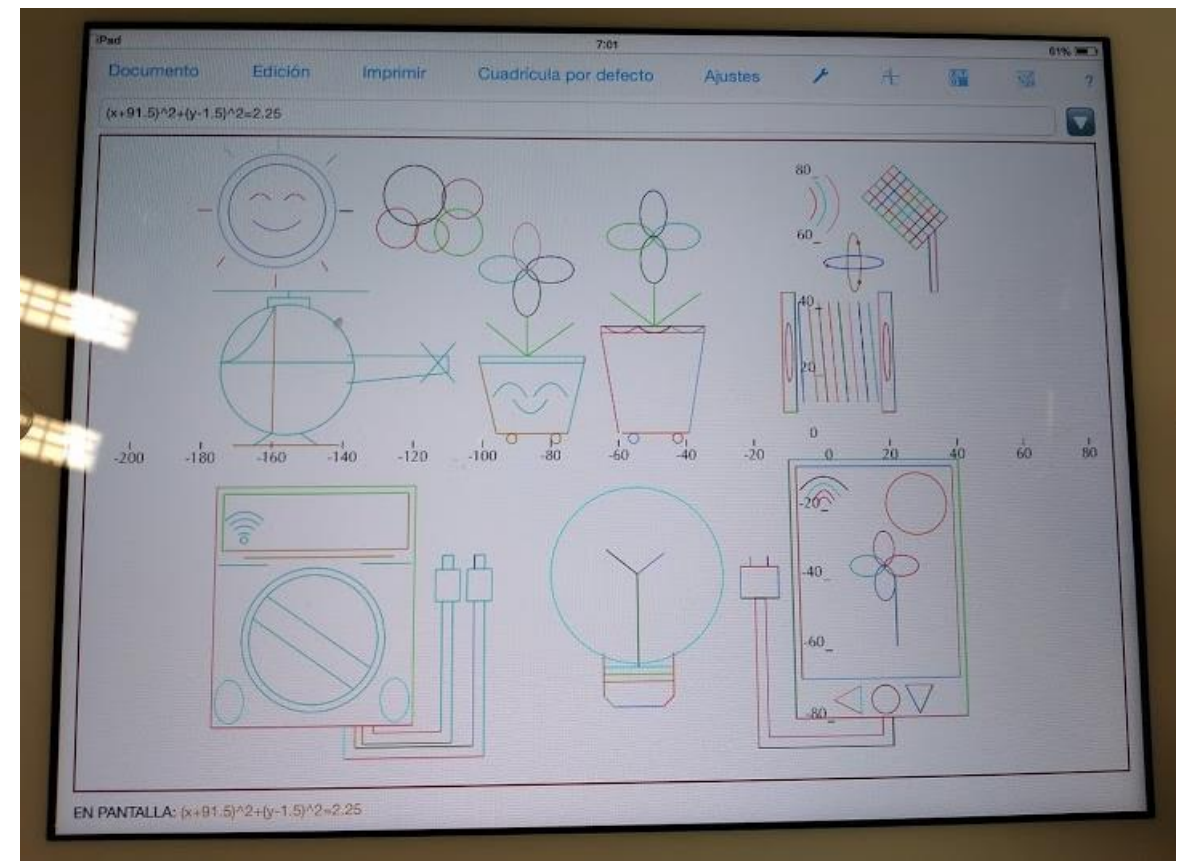

Figura 7. Imagen del paisaje expuesto en clase

Es importante mencionar que a lo largo de todo el proyecto, siempre se presentan diferentes obstáculos, errores que van desde la interpretación de cómo elaborar las tareas, fallas en la comunicación entre los integrantes del grupo, así como en la realización de las distintas actividades. Es por ello que se requiere una buena planeación, manejo de los tiempos y asesoría constante, principalmente para aquellos alumnos que se les dificulta mucho la asignatura, y como consecuencia, el desarrollo global del proyecto.

Es evidente que al principio a los jóvenes se les complica comprender todos los aspectos del proyecto que van a realizar, es difícil para ellos plasmar una idea en el papel, hasta concretarse en una imagen, la cual es creada a partir de ecuaciones matemáticas. Pero conforme van avanzando las diferentes etapas del proyecto, se van dispersando las dudas, se va mejorando la comprensión de cada una de estas, y al final se logra un claro entendimiento de la relación entre las imágenes elaboradas con las ecuaciones matemáticas respectivas, todo con el apoyo de GeoGebra.

El uso de GeoGebra en secuencias didácticas afines permite tanto a docentes como alumnos relacionar las bases teóricas con las herramientas digitales existentes.

Por todo lo anterior, se puede afirmar que esta actividad didáctica contribuyó a que el estudiante relaciona las diferentes ecuaciones vistas en el curso de geometría analítica con el desarrollo de su paisaje con el apoyo de la tecnología.

\section{Referencias}

Álvarez-Gayou, J. (2003). Cómo hacer investigación cualitativa: Fundamentos y Metodología. México: Paidós.

Biggs, J. (2006). Calidad del aprendizaje universitario. Madrid, España: Narcea.

Díaz, Frida y Hernández Gerardo. (2002) Estrategias docentes para un aprendizaje significativo. Una interpretación constructivista. 2da. Ed. Mc Graw Hill. México.

GeoGebra GmbH (2019). GeoGebra (versión ) [software] (2019) GeoGebra GmbH. https://www.geogebra.org/ 